\title{
Demonstration of Reduced Mitogenic and Osteoinductive Activities in Demineralized Allogeneic Bone Matrix from Vitamin D-deficient Rats
}

\author{
Russell T. Turner," John Farley, Jacob J. Vandersteenhoven," Sol Epstein," Norman H. Bell," and David J. Baylink \\ *Veterans Administration Medical Center, and Departments of Pharmacology and Medicine, Medical University of South Carolina, \\ Charleston, South Carolina 29403; ' Loma Linda University and Pettis Veterans Administration Hospital, Loma Linda, \\ California 92354; ' Department of Pathology, Medical University of South Carolina, Charleston, South Carolina 29425; \\ and "Albert Einstein Hospital, Philadelphia, Pennsylvania 19141
}

\begin{abstract}
Osteoinduction is the formation of ectopic bone that follows implantation of demineralized allogeneic bone matrix (DABM) and is believed to be secondary to the release of associated inductive factors from bone matrix. To clarify the role of vitamin D in osteoinduction, we implanted DABM from vitamin $D$-deficient rats ( $-D$ rats) into normal rats ( $+D$ rats). Because mitogens and osteocalcin might be involved in osteoinduction, these were measured. Mitogenic activity in extracts from mineralized allogeneic bone matrix (ABM) and DABM from both $+D$ and $-D$ rats was determined with an assay that utilizes monolayer cultures of embryonic chick calvarial cells. Osteocalcin in serum and DABM was measured by radioimmunoassay. DABM from -D rats did not promote osteoinduction as effectively as DABM from $+\dot{D}$ rats. Resorption of implant matrix from $-D$ rats was diminished compared with resorption of matrix from $+D$ rats $(P<0.01)$, and the decrease was attributed to a corresponding decrease in the number of osteoclasts in the implants $(P<0.02)$. Bone formation $(P<0.01)$ and total implant mineralization $(P<0.001)$ were significantly reduced in implants from $-D$ rats, and the reductions corresponded with a decline in the number of osteoblasts $(P<0.05)$. Mitogenic activity in DABM from $+D$ rats was only slightly decreased as compared with activity in $\mathrm{ABM}$, but DABM from $-D$ rats contained significantly less activity $(P<0.001)$. No mitogenic activity was identified in implants of DABM from either $+D$ or $-D$ rats 3 wk after implantation. Serum osteocalcin was significantly higher in $-D$ as compared with $+D$ animals. In contrast, the concentrations of osteocalcin in DABM from the two groups of animals were not significantly different from each other. These findings indicate that the diminished osteoinductive activity of DABM from -D rats results from deficiency of one or more mitogenic factors that are essential for inducing the proliferation and differentiation of bone cells at the implant site and that osteocalcin does not play a role in this regard.
\end{abstract}

\section{Introduction}

Bone induction by demineralized allogeneic bone matrix $(\mathrm{DABM})^{1}$ at heterotopic sites is ascribed to the inductive ef-

Dr. Turner's present address is GUGG.C007, Mayo Clinic, Rochester, MN 55905.

Received for publication 22 June 1987 and in revised form 4 January 1988

1. Abbreviations used in this paper: ABM, allogeneic bone matrix; DABM, demineralized allogeneic bone matrix; $-D$, vitamin $D$ deficient; $+\mathrm{D}$, vitamin $\mathrm{D}$ treated; $\mathrm{TdR}$, thymidine.

The Journal of Clinical Investigation, Inc.

Volume 82, July 1988, 212-217 fects of DABM on uncommitted mesenchymal cells causing them to pröliferate and differentiate toward an osteoblastic phenotype $(1,2)$. This phenomenon, which recapitulates the endochondral bone formation observed at osseous sites, is believed to result from release of mitogens from DABM. These mitogens were designated bone morphogenetic proteins (3-5) and bone-derived growth factors (2).

The role of calcium-regulating hormones, vitamin D, parathyroid hormone and calcitonin, and ions such as calcium and phosphate in mediating osteoinduction is not known. There is evidence that vitamin $D$ deficiency results in abnormalities in skeletal growth, remodeling, and mineralization (6). Consistent with these findings is the observation that osteoinduction is impaired when implants of DABM from normal rats are inserted into vitamin $D$-deficient rats ( $-D$ rats) $(7,8)$. Whereas defects in bone formation and bone resorption occur; the sequence of cellular events is not altered. Bone produced during vitamin $\mathrm{D}$ deficiency also was shown to be different biochemically. Rachitic collagen has increased hydroxylation of lysine $(9,10)$, altered cross-linking $(11,12)$, variable changes in the concentration and incorporation of glycosaminoglycans $(13,14)$, and changes in the content of phospholipid (8). Bone inductive proteins isolated from matrix produced in rachitic rats also were shown to be diminished (15). In view of these findings, the present studies were conducted to determine if defective osteoinduction seen in vitamin $\mathrm{D}$ deficiency is associated with diminished concentration or altered release of bone specific mitogens from bone matrix. Since synthesis of osteocalcin or Gla protein by osteoclasts is enhanced by 1,25 dihydroxyvitamin $\mathrm{D}_{3}\left[1,25(\mathrm{OH})_{2} \mathrm{D}_{3}\right](16,17)$, and since osteocalcin may be chemotactic for bone-forming cells (18), deficiency of osteocalcin could influence the osteoinductive response. Therefore, the concentration of osteocalcin in serum and in the implants also was determined.

\section{Methods}

Animals. Weanling Sprague-Dawley rats were obtained from Holtzman (Madison, WI). Donor animals which were used to make DABM for implants were fed ad libitum a semisynthetic diet which contained $0.6 \%$ calcium and $0.6 \%$ phosphorus (19). Half of the rats received a dietary supplementation of $2 \mathrm{IU}$ of vitamin $\mathrm{D}_{3}$ per gram of diet (+D rats), and the other half were raised in the dark and were fed the same diet but received no vitamin $\mathrm{D}$ ( $-\mathrm{D}$ rats). The animals were maintained on their respective diets for 3 mo. Recipient animals for the implants were raised on the same diet and received the same supplement of vitamin $\mathrm{D}_{3}$.

Preparation of allogeneic bone matrix and DABM. The DABM powder was prepared according to a modification of a protocol previously described (5). Femoral and tibial diaphyses from $+D$ and $-D$ rats were utilized. At least 16 animals were used for each preparation. Bones from $-D$ rats were grossly rachitic. There were shortening of the shafts, widening of the epiphyses, and increased pliability. The bones were stripped of soft tissue, crushed with a hammer, and rinsed in five changes of distilled water for $1 \mathrm{~h}$. The bone was then extracted with 
$100 \%$ ethanol (three changes in $1 \mathrm{~h}$ ) and subsequently extracted with chloroform/methanol 1:1 (three changes in $1 \mathrm{~h}$ ). Bone samples were dried overnight at $30^{\circ} \mathrm{C}$. The dried bone was crushed in a mill and sieved to a particle size ranging from 90 to $850 \mu \mathrm{m}$ in diameter. This allogeneic bone matrix (ABM) was decalcified at $25^{\circ} \mathrm{C}$ for $4 \mathrm{~h}$ with 0.5 $\mathrm{N} \mathrm{HCl}$ at $25 \mathrm{meq} / \mathrm{g}$ dried bone powder. After demineralization, the DABM was rinsed exhaustively in distilled water (10 changes in $2 \mathrm{~h}$ ), dehydrated in $100 \%$ ethanol (three changes in $1 \mathrm{~h}$ ), extracted in chloroform/methanol 1:1 (three changes in $1 \mathrm{~h}$ ), and dried overnight at $30^{\circ} \mathrm{C}$. The DABM was stored at $-70^{\circ} \mathrm{C}$. Before implantation, DABM was rinsed in sterile PBS. Nonosteoinductive DABM was prepared by heating DABM from $+\mathrm{D}$ rats at $100^{\circ} \mathrm{C}$ for $2 \mathrm{~h}$.

Implantation and analysis. DABM (20 mg) was inserted subcutaneously in thoracic and upper abdominal sites of $+D$ recipient rats. Preliminary experiments confirmed the importance of the site of implantation so that thoracic and upper abdominal and not lower abdominal sites were used (20). There were two DABM implants from $+D$ rats, two DABM implants from $-D$ rats, and one nonosteoinductive DABM implant from $+D$ rats in each animal. Ether was used as an anesthetic, and all surgical procedures were performed under aseptic conditions. Rats were killed 2, 3, and 10 wk after implantation. One implant from $a+D$ rat and one implant from $a-D$ rat were fixed in calcium acetate formalin, $\mathrm{pH}$ 6.8-7.0, decalcified, dehydrated, and embedded in JB-4 (Polysciences, Inc., Warrington, PA). 4- $\mu \mathrm{m}$ sections were stained with either toluidine blue or hematoxylin and eosin. The remaining implants were radiographed and then used for determination of calcium content and mitogenic activity. These samples were homogenized in $2 \mathrm{ml}$ of distilled water with a polytron probe and centrifuged at $500 \mathrm{~g}$. Precipitates were resuspended in $2 \mathrm{ml}$ of $1 \mathrm{~N} \mathrm{HCl}$ and were extracted for $48 \mathrm{~h}$ at $4^{\circ} \mathrm{C}$. After centrifugation at $500 \mathrm{~g}$, the solubilized extracts were stored at $4^{\circ} \mathrm{C}$ until further analysis. Calcium content of implants was measured by fluorometric titration assay (Calcette, Precision Systems, Inc., Sudbury, MA).

For analysis of osteocalcin in implants, $5 \mathrm{mg}$ of DABM each from $-D$ and $+D$ rats was extracted by incubation for $18 \mathrm{~h}$ at $4^{\circ} \mathrm{C}$ in a metabolic shaker in $1 \mathrm{ml} 0.5 \mathrm{M}$ EDTA Tris buffer, $\mathrm{pH}$ 6.5. After incubation, the mixture was centrifuged at $1,800 \mathrm{~g}$ for $10 \mathrm{~min}$ at $4^{\circ} \mathrm{C}$, and osteocalcin in the supernatant was analyzed by radioimmunoassay (see below).

Quantitative histomorphometric analysis. Randomized histologic samples were used for quantitative histomorphometric analysis of implants from $+D$ and $-D$ rats 3 and 10 wk after implantation. Residual implanted bone matrix (old bone matrix), new bone matrix, forming surface, and resorbing surface were measured. Residual or old implant matrix, the remnant of the original donor implant matrix, was identified as acellular collagenous matrix. The new bone formed during the inductive response was a cellular collagenous matrix which was bordered by cement lines and usually showed osteoblasts and resorptive cells on free surfaces. The forming surface was identified by a layer of osteoblasts along any free surface of residual and new bone matrix. The cells were identified as having a cuboidal shape and prominent Golgi apparatus situated between the nucleus and the base. Resorbing surfaces were determined by the presence of multinucleated cells along the free matrix surfaces or mononuclear cells within prominent resorption lacunae on free surfaces of new and residual bone matrix.

Bone measurements were made with a model BH-2 microscope system (Olympus Corp. of America, New Hyde Park, NY): the tissue section image was projected with a video camera (SMI-Unicomp, Atlanta, GA) onto a high-resolution video screen interphased with a $\mathrm{Hi}$ Pad digitizer board (Houston Instruments, Houston, TX) and Apple Ile computer. The mean values for each section were calculated from measurements of five fields $\left(49,000 \mu \mathrm{m}^{2}\right.$ per field $)$ at the 3-wk interval and four fields $\left(196,000 \mu \mathrm{m}^{2}\right.$ per field) at the 10 -wk interval. Since there were no active forming or resorbing surfaces at $10 \mathrm{wk}$, measurements were made at a lower magnification than otherwise necessary to reliably identify bone cells. Statistical analysis was determined from the number of individual implants measured and not from the number of sections examined. Values are expressed as micrometer (forming and resorbing certain lengths) and square micrometer (implant matrix and new bone matrix)/49,000- $\mu \mathrm{m}^{2}$ field.

Mitogenic activity. Samples of ABM, DABM from $-\mathrm{D}$ and $+\mathrm{D}$ rats, and implants of DABM recovered 3 wk after implantation were transferred to dialysis tubing (nominal cutoff: $12,000-14,000 \mathrm{~mol} \mathrm{wt}$ )

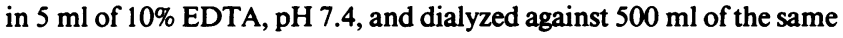
EDTA solution for $7 \mathrm{~d}$ at $4^{\circ} \mathrm{C}$ with continuous stirring. The soluble extracts were then removed from the dialysis tubing, separated from the organic residues (primarily collagen) by centrifugation, and individually dialyzed against 2 liters of $5 \mathrm{mM}$ phosphate buffer for $14 \mathrm{~d}$ with a minimum of six changes of the buffer.

The dialyzed extracts were concentrated by lyophilization and were sterilized by microfiltration (Gelman Sciences, Inc., Ann Arbor, MI) (21). Protein was determined by the binding method of Bradford (22). Mitogenic activity was determined by stimulation of $\left[{ }^{3} \mathrm{H}\right]$ thymidine $\left(\left[{ }^{3} \mathrm{H}\right] \mathrm{TdR}\right)$ incorporation into TCA-precipitable material in monolayer cultures of chick calvarial cells (23). All samples were tested at protein concentrations ranging from 0.1 to $200 \mu \mathrm{g} / \mathrm{ml}$ of culture medium (DME, $70 \times 10^{3}$ cells, in $1 \mathrm{ml}$ per 24-place multiwell or $35 \times 10^{3}$ cells in $0.5 \mathrm{ml}$ per 48 -place multiwell), and incorporation of $\left[{ }^{3} \mathrm{H}\right] \mathrm{TdR}$ was assessed during a $2-\mathrm{h}$ pulse beginning $18 \mathrm{~h}$ after addition of the extract material. Previous studies established that the increased incorporation of $\left[{ }^{3} \mathrm{H}\right] \mathrm{TdR}$ in this assay system in response to EDTA extracts of human, bovine, rat, and chicken bone, reflects an increase in cell number (23-25). Bovine serum albumin was included as a control for the effect of adding protein to the protein-free medium. Similar results were obtained with human serum albumin, ovalbumin, or rat serum albumin as controls (data not shown). Activity was expressed as percentage of control counts per minute ( $n=6$ culture wells for each tested dose of effector, and $n=12-18$ for controls). One unit of mitogenic activity is defined as the amount of effector required to increase the incorporation of $\left[{ }^{3} \mathrm{H}\right] \mathrm{TdR}$ by $100 \%$ above control values. Activities determined for each effector in the dose-response studies were normalized for protein, so that all values could be expressed as units per milligram of protein. Results are reported as mean \pm SD for all doses of effector within the linear range of the dose-response curve.

There are two major sources of variations in these assays: those between different preparations of calvarial cells and those between different configurations of tissue culture plate.

First, the primary cultures of embryonic chick calvarial cells used in these assays were prepared at weekly intervals, and significant variations in the magnitude of the response to effectors was seen with different cell preparations. This finding may be due to variations in the ratio of progenitor cells to differentiated osteoblasts. For example, the mitogenic response to the addition of $1 \%$ fetal calf serum, included as a positive control, varied between 430 and $1,180 \%$ of control in the cell preparations used for these investigations. Because this variation in response was large between cell preparations but relatively small within a given cell preparation, that is, the coefficient of variation within cell preparations ranged from $9 \%$ to $21 \%$, results are expressed from studies which use the same cell preparation. All observations, however, were confirmed by studies with at least two cell preparations. Second, the absolute value of the mitogenic response of the cells was also different in 48-place multiwell plates as compared to 24-place multiwell plates. During the course of these studies we adapted our assays to 48-place plates so that some of the data were obtained with each system. However, we have made comparisons only in studies from a single preparation of cell and a single 24- or 48-place system.

Analytical procedures. Serum calcium was measured by fluorometric titration assay (Calcette). Protein in DABM was measured by the dye-binding method of Bradford (22). Serum 25-hydroxyvitamin D (25-OHD) was measured after extraction by a protein-binding assay with serum from $-\mathrm{D}$ rats $(26) .1,25(\mathrm{OH})_{2} \mathrm{D}$ was measured after extraction and purification by high performance liquid chromatography with a receptor-binding assay which utilizes cytosol from the small intestine of vitamin D-deficient chicks (26). Osteocalcin in serum and extracts of DABM was determined in duplicate in aliquots of $100 \mu \mathrm{l}$ and extracts of $5 \mathrm{mg}$, respectively, by radioimmunoassay as previously 


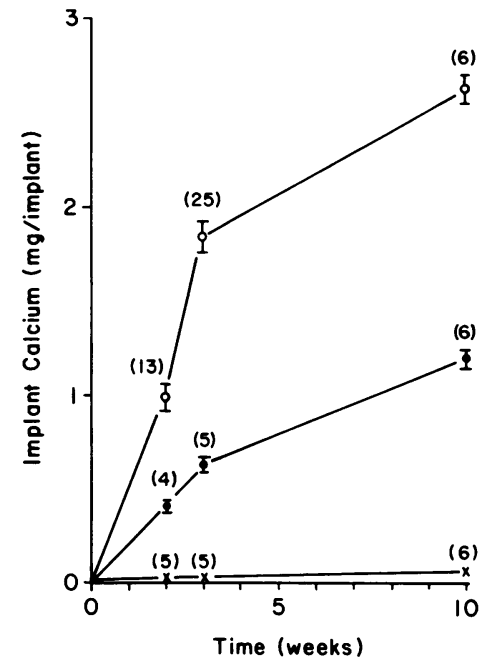

Figure 1. Calcium content of implants of DABM from vitamin D-treated $(+D)$ rats (O), vitamin D-deficient $(-D)$ rats $(\bullet)$, and of heat-treated nonosteoinductive DABM from $+\mathrm{D}$ rats $(x)$. The number in brackets is the number of observations. Results are given as mean $\pm \mathrm{SD}$.

described (27). Osteocalcin in DABM was measured in $1 \mathrm{ml}$ of Tris buffer, $\mathrm{pH}$ 6.5. Results are expressed as nanogram per milliliter for serum and nanogram per milligram of bone for DABM.

\section{Results}

The $-D$ rats were hypocalcemic and had a serum calcium of $<6.0 \mathrm{mg} / \mathrm{dl}$. Serum 25-OHD was below the detection limit of $0.5 \mathrm{ng} / \mathrm{ml}$, and serum $1,25(\mathrm{OH})_{2} \mathrm{D}$ was below the detection limit of $5 \mathrm{pg} / \mathrm{ml}$, indicating that the animals were severely vitamin D deficient. Implants of DABM from $-D$ rats which had been inserted in $+D$ rats showed reduced calcium content at all time points when compared to implants of DABM from +D rats in the same host animals (Fig. 1).

Qualitative examination of stained sections from implants recovered 2 wk after implantation revealed similar morphologies in $D A B M$ from $+D$ rats and $-D$ rats. At this stage the implants were surrounded by a well-organized fibrous capsule. The implant bone matrix particles were largely intact, and there was relatively little new bone matrix. At 3 wk after implantation, the implants from $+D$ rats displayed an extensive network of resorption cavities. These cavities were filled with hematopoietic marrow cells and their surfaces were lined with active osteoblasts and osteoclasts. Gross radiographs of the implants revealed extensive radiodense deposits that were uniformly distributed throughout the implants. In contrast, DABM from -D rats showed no large resorption cavities, little or no hematopoietic cells, and isolated bone surfaces covered with differentiated bone cells. Radiographs of these implants revealed a marked absence of radiodense deposits. By 10 wk after implantation, DABM from $+D$ rats had large central cavities filled with fatty marrow. Although the cavities were bordered by extensive deposits of new bone, the bone surfaces were essentially inactive. By $10 \mathrm{wk}$ after placement, implants from $-D$ rats appeared similar to those obtained at 3 wk for that group except that no differentiated bone cells were observed.

In Table I, quantitative measurements of the residual bone matrix area, new bone matrix area, forming surface length, and resorbing surface length in implants from $+D$ rats and $-D$ rats are compared from samples obtained 3 and 10 wk after implantation. At both times, there was significantly more residual bone matrix in implants from $-D$ rats. Furthermore, in the interval from 3 to $10 \mathrm{wk}$ after implantation, the residual bone matrix had decreased significantly in DABM from $+\mathrm{D}$ rats, whereas there was no decrease in residual matrix in DABM from $-D$ rats. There were similar amounts of newly formed bone matrix in both types of implants $3 \mathrm{wk}$ after implantation. The newly formed bone in implants of DABM from $+D$ rats had increased significantly during the interval from 3 to 10 wk after implantation. In contrast, no newly formed bone matrix was found in implants from $-D$ rats 10 wk after implantation. Presumably the amount of new bone matrix present at $3 \mathrm{wk}$ was subsequently resorbed. At 3 wk after implantation, the amount of resorbing surface of implants from $+D$ rats was much greater than that of implants from $-D$ rats. No resorbing surface was found in implants from either group 10 wk after implantation. Similarly, 3 wk after insertion the forming surface in implants from $+D$ rats was greater than that of implants from $-D$ rats, and by 10 wk no forming surface was observed in either group.

The values for mitogenic activity in extracts of DABM and $A B M$ from $+D$ and $-D$ rats are summarized in Tables II and III. In the two studies summarized in Table II, $1 \%$ fetal calf serum stimulated incorporation of $\left[{ }^{3} \mathrm{H}\right] \mathrm{TdR}$ to 480 and $540 \%$ compared to that of controls, whereas in the study summarized in Table III, $1 \%$ fetal calf serum increased the incorpora-

Table I. Quantitative Histomorphometry of Implants 3 and 10 wk after Implantation

\begin{tabular}{llccc}
\hline \multicolumn{1}{c}{ Measurement } & Unit & DABM from + D rats & DABM from - D rats & $P$ value \\
\hline 3 wk & & & & \\
Residual matrix area & $\mu \mathrm{m}^{2}$ & $15,800 \pm 1,700$ & $34,350 \pm 5,000$ & $<0.025$ \\
New bone matrix area & $\mu \mathrm{m}^{2}$ & $3,400 \pm 1,500$ & $2,300 \pm 1,650$ & $110 \pm 60$ \\
Forming surface length & $\mu \mathrm{m}$ & $300 \pm 90$ & $30 \pm 15$ & $<0.05$ \\
Resorbing surface length & $\mu \mathrm{m}$ & $240 \pm 40$ & $44,000 \pm 2,700$ \\
10 wk & & & ND & ND \\
Residual matrix area & $\mu \mathrm{m}^{2}$ & $7,650 \pm 4,750^{*}$ & ND \\
New bone matrix area & $\mu \mathrm{m}^{2}$ & $23,800 \pm 4,900^{\ddagger}$ & $\mathrm{ND}$ & $<0.01$ \\
Forming surface length & $\mu \mathrm{m}$ & $\mathrm{ND}$ & &
\end{tabular}

Results are presented as mean \pm SD of four or five observations. DABM was obtained from vitamin D-treated $(+D)$ and vitamin $D$-deficient $(-D)$ animals and implanted into $+D$ animals. ND, not detected. ${ }^{*} P<0.05$ vs. values at 3 wk. ${ }^{\ddagger} P<0.01$ vs. values at 3 wk. 
Table II. Comparison of Mitogenic Activity in Extracts of DABM before and after Implantation

\begin{tabular}{|c|c|c|c|}
\hline \multirow[b]{2}{*}{ Experiment } & \multirow[b]{2}{*}{ Treatment } & \multicolumn{2}{|c|}{ Activity* } \\
\hline & & Protein & Bone \\
\hline & & $U / m g$ & $U / m g$ \\
\hline \multirow[t]{3}{*}{1} & DABM from $+D$ rats & $204 \pm 38$ & $13.6 \pm 2.5$ \\
\hline & DABM from $-D$ rats & $83 \pm 13$ & $4.5 \pm 0.7$ \\
\hline & $P$ value & $<0.01$ & $<0.02$ \\
\hline \multirow[t]{3}{*}{2} & $\mathrm{DABM}$ from $+\mathrm{D}$ rats & $202 \pm 16$ & $13.5 \pm 2.5$ \\
\hline & DABM from $-D$ rats & $65 \pm 13$ & $3.5 \pm 0.7$ \\
\hline & $P$ value & $<0.01$ & $<0.01$ \\
\hline
\end{tabular}

Results are given as mean \pm SD of six observations. Two separate assays were performed with 24-well plates.

DABM was obtained from vitamin D-treated (+D) and vitamin Ddeficient $(-D)$ animals.

* No activity was detected in implants from $+\mathrm{D}(n=5)$ or $-\mathrm{D}$ rats $(n=6) 3$ wk after implantation.

tion of $\left[{ }^{3} \mathrm{H}\right] \mathrm{TdR}$ to $870 \%$ that of control. This indicates that the cell preparation used for the latter study was more responsive.

The results in Table II were obtained with 24-place plates, and those in Table III were obtained with 48-place plates. Although direct comparison is not possible, separate studies revealed that when cells from a single preparation were used the mitogenic response in an extract of DABM from $+D$ rats averaged $204 \mathrm{U} / \mathrm{mg}$ protein in 24-place plates and $516 \mathrm{U} / \mathrm{mg}$ protein in 48-place plates. However, the observed ratio of activity in extracts of $D A B M$ from $+D$ rats and $-D$ rats was not different regardless of whether 24-place or 48-place plates were used.

DABM from -D rats had a significantly lower content of mitogenic activity than DABM from $+D$ animals (Table II). $<15 \%$ additional mitogenic activity was obtained when DABM from $+D$ or $-D$ rats was reextracted (data not shown). This indicates that extraction was equally efficient in both experimental groups. There was significantly less activity in extracts of $A B M$ from $-D$ than from $+D$ rats (Table III). A reduction of $20 \%$ was observed in mitogenic activity in ex-

Table III. Comparison of Mitogenic Activity in Extracts from $A B M$ and $D A B M$

\begin{tabular}{ccc}
\hline & \multicolumn{2}{c}{ Activity } \\
\cline { 2 - 3 } Treatment & Protein & Bone \\
\hline & $U / m g$ & $U / m g$ \\
ABM bone from +D rats & $1,420 \pm 52$ & $87.7 \pm 3.1$ \\
ABM bone from $-D$ rats & $470 \pm 28^{*}$ & $21.2 \pm 2.6^{*}$ \\
DABM from +D rats & $1,060 \pm 42$ & $70.9 \pm 2.8$
\end{tabular}

Results are presented as mean \pm SD of six observations. Assays were performed with 48-well plates. ABM was obtained from vitamin Ddeficient $(-D)$ and vitamin D-treated $(+D)$ rats. DABM was obtained from vitamin $D$-treated $(+D)$ rats. Note that there was a reduction of $20 \%$ in mitogenic activity in preparation of DABM from ABM (see Methods).

$* P<0.001$ vs. values for $\mathrm{ABM}$ from $+\mathrm{D}$ rats. tracts of DABM as compared to extracts of $A B M$. These findings indicate that the diminished activity in DABM from $-D$ rats does not represent a loss in activity caused by preparation of the demineralized matrix but is a property of bone from $-D$ rats. Further, mitogenic activity was not detected in implants of DABM from $+D$ or $-D$ rats 3 wk after implantation (Table II). These results indicate that the mitogenic activity is lost during this interval. The mitogenic activity of DABM was much greater than the activity of bovine serum albumin when the latter was tested at very high concentrations of protein (Fig. 2).

Serum osteocalcin was significantly higher in the $-D$ than in the $+\mathrm{D}$ animals after $12 \mathrm{wk}$ on the respective diets, $98 \pm 5$ ( $n$ $=18)$ vs. $80 \pm 4(n=17) \mathrm{ng} / \mathrm{ml} P<0.02$, respectively. However, the concentration of osteocalcin in extracts of the DABM from $-\mathrm{D}$ and $+\mathrm{D}$ rats was not different, $15.3 \pm 5.6$ vs. $12.4 \pm 2.9$ $\mathrm{ng} / \mathrm{mg}$ of bone $(n=3)$, respectively.

\section{Discussion}

After implantation of DABM, osteoinduction recapitulates endochondral bone formation in a consistent sequence of cellular events $(5,28,29)$. These include transitory periods of cartilage synthesis, resorption of implant matrix, formation and resorption of new matrix as well as continued resorption of implant matrix and finally, formation of marrow in resorption cavities. The net result of these remodeling processes is the transformation of the relatively homogenous implant to an ossicle consisting of a thin shell of compact bone surrounding a large central cavity filled with mature fatty marrow.

As compared to implants of DABM from $+\mathrm{D}$ rats, implants of DABM from $-D$ rats had less resorption of the implant matrix, less formation of new bone matrix, and less marrow formation. By 10 wk after implantation, remodeling

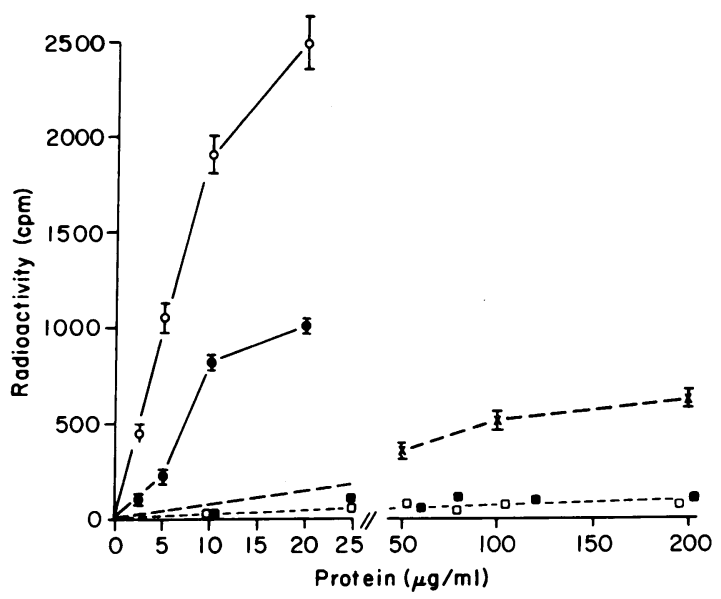

Figure 2. Incorporation of $\left[{ }^{3} \mathrm{H}\right] \mathrm{TdR}$ into embryonic chick calvarial cells as a function of protein content. Implants of DABM from vitamin D-treated $(+D)$ rats $(0)$, and vitamin D-deficient $(-D)$ rats $(\bullet)$, and implants of DABM from $+\mathrm{D}$ rats $3 \mathrm{wk}$ after implantation (口), or implants of DABM from $-D$ rats 3 wk after implantation $(\square)$ were extracted. Results are compared to those obtained with purified bovine serum albumin $(x)$. Radioactivity is expressed as cpm incorporated by experimental cultures - cpm incorporated by cultures incubated with protein-free media. Results are given as mean \pm SD of six observations. 
had ceased in both types of implants. The diminished resorption of implant matrix and diminished formation of new bone matrix were directly attributable to a reduction in the number of osteoclasts and osteoblasts, respectively. The defect in marrow formation in the implants from $-D$ animals may be due to the failure to form resorption cavities and possibly to impaired differentiation of marrow cells.

We previously reported that osteoinduction is greatly reduced in magnitude when DABM from $+\mathrm{D}$ rats is implanted into $-D$ animals (7). This finding was confirmed in a subsequent study (15). Since the initial events of osteoinduction, formation of fibrous capsule, penetration of blood vessels, chondrogenesis, and non-bone cell-mediated resorption of implants appeared normal, we proposed that the defect might be in the propagation of the inductive response and perhaps in the recruitment of progenitor cells necessary for subsequent differentiation into bone cells.

The present studies demonstrate that DABM from $-D$ rats implanted into normal animals does not promote osteoinduction as effectively as DABM from $+D$ rats. Thus, the defect must reside in the bone matrix from the donor rats. Similar results were reported by other investigators (15). The defect appears to be in the amount or activity of specific factor(s) required for osteoinduction rather than in nonspecific changes in the chemistry of rachitic bone matrix such as abnormal cross-linking of collagen.

Additional evidence to support a role for vitamin D in the regulation of noncollagenous bone matrix proteins is the demonstration that osteocalcin, or Gla protein, the most abundant noncollagenous component of bone matrix, is chemotactic for human and mouse peripheral monocytes and cultured rat osteosarcoma cells which have a number of characteristics of the osteoblast (18). Modulation of osteocalcin by vitamin $\mathrm{D}$ is demonstrated by the striking reductions of osteocalcin in chick and rat bone in vitamin $D$ deficiency $(30,31)$. A role for vitamin $D$ is further supported by the observation that $1,25(\mathrm{OH})_{2} \mathrm{D}_{3}$ stimulates secretion of osteocalcin by osteosarcoma cells in culture (16), and increases circulating immunoreactive osteocalcin in normal rats (17). In contrast to previous findings (31), serum osteocalcin was higher in -D as compared to $+\mathrm{D}$ animals in the present study. Further, values for osteocalcin in the DABM from $+D$ and $-D$ animals before implantation were not significantly different from each other.

The values for osteocalcin in implants of bone from $-D$ and $+\mathrm{D}$ rats were quite low. Previous studies demonstrated that osteocalcin normally makes up from $1-3 \%$ of rat bone and that osteocalcin is removed by demineralization with formic acid $(32,33)$. The fact that demineralized material was used for the implants in the present study accounts for the low values of osteocalcin. These findings indicate that decreases in osteocalcin did not contribute to defective osteoinduction in implants from $-D$ rats. In this regard, warfarin was shown to deplete osteocalcin in rat bone, and osteoinduction was not altered when implants of warfarin-treated animals were utilized (33).

Differences in protocol and age of the animal at the time the vitamin D-deficient diet was started may be responsible for the lack of a decrease of osteocalcin in serum and bone of our $-\mathrm{D}$ rats. The occurrence of decreases in the concentration of osteocalcin in bone and the circulation reported by other investigators is related to duration of the $-D$ diet (30) and may be related to the age at which the diet was begun. It is possible that the values would have declined in our rats had they been started at an earlier age or been kept on the $-D$ diet for a longer period of time.

Osteoinduction requires the invasion and population of implants by host cells. Precursor cells proliferate and differentiate into osteoblasts, osteocytes, and osteoclasts. These processes are regulated, at least in part, by factors released from the implants (2-5). It is therefore of considerable interest that the extractable mitogenic activity of DABM from $-D$ rats was dramatically less than that of DABM from $+D$ rats. This defect appears to be in the amount of mitogenic activity rather than altered diffusion of the active principle(s) during preparation because the ratio of the mitogenic content of $A B M$ from $-D$ rats compared to $A B M$ from $+D$ rats was similar to the ratio of mitogenic content in DABM from $-D$ rats and in DABM from $+D$ rats. There was a modest decrease in mitogenic activity during preparation of DABM from intact bone. All of the activity in implants of DABM from $-D$ rats as well as activity in implants from $+D$ rats was lost by 3 wk after implantation.

A number of growth factors are produced by rat bone cells (34) including transforming growth factor- $\beta$-like activity (35), $\beta_{2}$-microglobulin (36), and insulinlike growth factor I or somatomedin $C$ (37). In addition, skeletal growth factor and transforming growth factor- $\beta$-like activity were found in extracts of bovine bone matrix (25) and skeletal growth factor was present in abstracts of human bone (24). The component(s) responsible for the mitogenic activity in DABM in the present studies has not been identified.

The results of the present studies demonstrate that bone matrix from $-D$ animals is less effective in promoting osteoinduction and contains less mitogenic activity. Whether this mitogenic activity plays a role in osteoinduction remains to be determined.

\section{Acknowledgments}

The authors wish to acknowledge the expert assistance of Ms. Sheryl Shaw for the assays of 25-hydroxyvitamin D and Mrs. Allison Joseph for preparation of this manuscript. These studies were supported by the Veterans Administration and grant AM-33025 from the National Institutes of Health. Norman $\mathrm{H}$. Bell was a Veterans Administration Medical Investigator.

\section{References}

1. Urist, M. R. 1979. Bone morphodifferentiation and tumorigenesis. Perspect. Biol. Med. 2:S89-S113.

2. Urist, M. R., R. J. DeLange, and G. A. M. Finerman. 1983. Bone cell differentiation and growth factors. Science (Wash. DC). 220:680686.

3. Urist, M. R., T. A. Dowell, P. H. Hay, and B. S. Strates. 1968. Inductive substrates for bone formation. Clin. Orthop. Relat. Res. 59:59-96.

4. Amitani, K., and Y. Nakata. 1975. Bone induction by lyophilized osteosarcoma cells in mice. Calcif. Tissue Res. 17:139-150.

5. Reddi, A. H., and C. B. Huggins. 1972. Biochemical sequences in transformation of normal fibroblasts in adolescent rats. Proc. Natl. Acad. Sci. USA. 69:1601-1605.

6. Baylink, D. J., E. R. Morey, J. L. Ivey, and M. E. Stauffer. 1980. Vitamin D in bone. In Vitamin D: Molecular Biology and Clinical Nutrition. A. W. Norman, editor. Marcel Dekker, Inc. New York. 387-453. 
7. Vandersteenhoven, J. J., R. T. Turner, N. H. Bell, and F. A. DeLustro. 1983. The role of vitamin D in osteoinduction. Calcif. Tissue Int. 35:704. (Abstr.)

8. Wuthier, R. E. 1971. Zonal analysis of phospholipids in epiphysial cartilage and bone of normal and rachitic chickens and pigs. Calcif. Tissue Res. 8:36-53.

9. Barnes, M. J., B. L. Constable, L. F. Morton, and E. Kodicek. 1973. Bone collagen metabolism in vitamin D deficiency. Biochem. J. 132:113-115.

10. Toole, B., A. H. King, R. L. Trelstad, and J. Gross. 1972. Collagen heterogeneity within different growth regions of long bones of rachitic and non-rachitic chicks. Biochem. J. 127:715-720.

11. Mechanic, G. L., S. N. Toverud, and W. K. Ramp. 1972. Quantitative changes of bone collagen crosslinks and precursors in vitamin D deficiency. Biochem. Biophys. Res. Commun. 47:760-765.

12. Mechanic, G. H., S. U. Toverud, W. K. Ramp, and W. A. Gonnerman. 1975. The effect of vitamin D on the structural crosslinks and maturation of chick bone collagens. Biochim. Biophys. Acta. 393:419-425.

13. Dziewiatkowiski, D. D. 1954. Vitamin D and endochondral ossification in the rat as indicated by the use of sulphur-35 and phosphorus-32. J. Exp. Med. 100:25-32.

14: Deiss, W. P., Jr., and D. L. Hern. 1979. Bone matrix studies. Influences of parathyroid extract, calcitonin, and cholecalciferol and of rickets and its treatment. Biochim. Biophys. Acta. 584:311-326.

15. Sampath, T. K., S. Weintraub, and A. H. Reddi. 1984. Extracellular matrix proteins involved in bone induction are vitamin D dependent. Biochem. Biophys. Res. Commun. 124:829-835.

16. Price, P. A., and S. A. Baukol. 1980. 1,25-Dihydroxyvitamin $D_{3}$ increases synthesis of the vitamin K-dependent bone protein by osteosarcoma cells. J. Biol. Chem. 255:11660-11663.

17. Price, P. A., and S. A. Baukol. 1981. 1,25-Dihydroxyvitamin $D_{3}$ increases serum levels of the vitamin K-dependent bone protein. Biochem. Biophys. Res. Commun. 99:928-935.

18. Mundy, G. R., and J. W. Poser. 1983. Chemotactic activity of the $\gamma$-carboxyglutamic acid containing protein in bone. Calcif. Tissue Int. 35:164-168.

19. Stauffer, M., D. J. Baylink, J. Wergedal, and C. Rich. 1973. Decreased bone formation, mineralization, and enhanced resorption in calcium deficient rats. Am. J. Physiol. 225:269-276.

20. Reddi, A. H., and C. B. Huggins. 1975. Formation of bone marrow in fibroblast-transformation ossicles. Proc. Natl. Acad. Sci. USA. 72:2212-2216.

21. Puzas, J. E., R. H. Drivdahl, G. A. Howard, and D. J. Baylink. 1981. Endogenous inhibitor of bone cell proliferation. Proc. Soc. Exp. Biol. Med. 166:113-122.

22. Bradford, M. M. 1976. A rapid and sensitive method for the quantitation of microgram quantities of protein utilizing the principle of protein-dye binding. Anal. Biochem. 72:248-254.

23. Drivdahl, R. H., J. E. Puzas, G. A. Howard, and D. J. Baylink.
1981. Regulation of DNA synthesis in chick calvaria cells by factors from bone organ culture. Proc. Soc. Exp. Biol. Med. 166:113-122.

24. Farley, J. R., T. Masuda, J. E. Wergedal, and D. J. Baylink. 1982. Human skeletal growth factor: characterization of the mitogenic effect on bone cells in vitro. Biochemistry. 21:3508-3513.

25. Linkhart, T. A., J. C. Jennings, S. Mohan, G. K. Wakely, and D. J. Baylink. 1986. Characterization of mitogenic activities extracted from bovine bone matrix. Bone. 7:479-487.

26. Lambert, P. W. 1983. Assay for multiple vitamin D metabolites. In Assay of Calcium Regulating Hormones. D. Bickle, editor. Springer-Verlag, New York. 99-124.

27. Jowell, P. S., S. Epstein, M. D. Fallon, T. A. Reinhardt, and F. Ismail. 1987. 1,25-Dihydroxyvitamin $\mathrm{D}_{3}$ modulates glucocorticoidinduced alteration in serum bone gla protein and bone histomorphometry. Endocrinology. 120:531-536.

28. Urist, M. R. 1965. Bone formation by autoinduction. Science (Wash. DC). 150:893-899.

29. Vandersteenhoven, J. J., and M. Spector. 1983. Histological investigation of bone induction by demineralized allogeneic bone matrix: a natural biomaterial for ossedus reconstruction. J. Biomed. Mater. Res. 17:1003-1013.

30. Lian, J. B., M. J. Glimcher, A. H. Rafosse, P. V. Hauschka, P. M. Gallop, L. Cohen-Solal, and B. Reit. 1982. Alterations of the $\gamma$-carboxyglutamic acid and osteocalcin concentrations in vitamin Ddeficient chick bone. J. Biol. Chem. 257:4999-5003.

31. Lian, J. B., D. L. Carnes, and M. J. Glimcher. 1987. Bone and serum concentrations of osteocalcin as a function of 1,25-dihydroxyvitamin $D_{3}$ circulating levels in bone disorders in rats. Endocrinology. 120:2123-2130.

32. Price, P. A., J. W. Lothringer, and S. K. Nishimoto. 1980. Absence of the vitamin k-dependent bone protein in fetal rat mineral: evidence for another $\gamma$-carboxyglutamic acid-containing component in bone. J. Biol. Chem. 255:2938-2942.

33. Price, P. A., and M. K. Williamson. 1981. Effects of warfarin on bone: studies on the vitamin k-dependent protein of rat bone. J. Biol. Chem. 256:12754-12759.

34. Canalis, E., W. A. Peck, and L. G. Raisz. 1980. Stimulation of DNA and collagen synthesis by autologous growth factor in cultured fetal rat calvariae. Science (Wash. DC). 210:1021-1023.

35. Centralla, M., and E. Canalis. 1987. Isolation of EGF-dependent transforming growth factor (TGF $\beta$-like) activity from culture medium conditioned by fetal rat calvariae. J. Bone Mineral Res. 2:29-36.

36. Canalis, E., T. McCarthy, and M. Centralla. 1987. A bone-de-

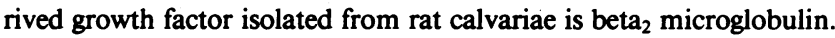
Endocrinology. 121:1198-1200.

37. Stracke, H., A. Schulz, D. Moeller, S. Russell, and H. Schatz. 1984. Effect of growth hormone on osteoblast and demonstration of somatomedin C/IGF I in bone organ culture. Acta Endocrinol. 107:16-24. 